England. This report has been referred to by Commissioning Groups when reviewing and commissioning services.

The information produced by ChiMat is based on data from the hospital's coding department, who determine the admission reason from discharge letters completed by junior doctors.

We carried out a retrospective audit to review:

- The accuracy of coding

- Medical management of Paediatric Emergency Epilepsy admissions.

Methods We reviewed the medical notes of all patients coded as having a Paediatric Emergency Epilepsy admission during 2011. There were 78 patients during this period, and information was collected using a standardised proforma. Inappropriately coded patients were excluded from analysis in the second part of the audit.

Results 10 of the 78 patients (12.8\%) were exclusively under the care of the adult physicians, with age range $16-19$ years. Of the other 68 patients, 15 were incorrectly coded (22\%).

Review of the medical management in the remaining 53 admissions, showed areas for improvement in medication adherence, patient education and awareness, and community management plans.

Conclusions This study has shown the importance of accurate data coding, as this is used to review the service we provide, highlighting exceptional practice as well as areas which require improvement. Variation in practice and value in healthcare are the current quality indicators which are used, to compare hospitals and clinicians, and to continue the quality improvement cycle. It is therefore in the interest of all to engage with clinical coding to ensure accurate, robust data is being used.

Our audit has led to more streamlined management of patients with epilepsy, including the consideration of more community-based management plans and proposal for a Paediatric Epilepsy Specialist Nurse. There has also been quality improvement effects, including introduction of a weekly epilepsy-related admissions report which is reviewed for accuracy, monthly epilepsy peer-review meeting to review all admissions and challenging cases, importance of accurate coding on discharge letters being emphasised to junior doctors at induction, and introduction of a checklist for management of patients with epilepsy.

\section{G69(P) NARCOLEPSY - AN IMPORTANT BUT RARE PAEDIATRIC DIAGNOSIS}

A Ratcliffe, C Kallappa. Paediatric Department, Good Hope Hospital, Sutton Coldfield, UK

\subsection{6/archdischild-2015-308599.68}

Aim To present patients investigated for narcolepsy in order to increase awareness of its clinical features.

Methods 5 patients referred with possible narcolepsy to the sleep clinic.

Results

1. 8 year old boy with excessive daytime sleepiness and cataplexy. HLA typing has shown HLA- DQB*06:02. MSLT showed short sleep latency. Awaiting lumbar puncture for CSF analysis of orexin level. Commenced on methylphenidate with good response.

2. 6 year old boy with 4 year history of falling asleep quickly including very short distances in the car, at school, and before finishing meals. HLA typing is positive for DQB1*06-02. Commenced on methylphenidate.
3. 13 year old boy with 5 year history of falling asleep several times a day often missing his bus stop and has recurrent naps in school. Commenced on methylphenidate.

4. 13 year old girl who falls asleep in lessons however related to boredom rather than irresistable desire to fall asleep. Found to be anaemic. Commenced on iron supplementation.

5. 8 year old girl initially presenting with excessive daytime sleepiness with hallucinations on sleeping and waking. She was HLA DQB*06-02 positive suggesting narcolepsy with hypnagogic and hypnopompic hallucinations. MSLT not diagnostic of narcolepsy but had epileptiform changes on EEG. Commenced on clonazepam with improvement in hallucinations.

Conclusion Narcolepsy is an autoimmune neurological disorder characterised by excessive daytime sleepiness associated with cataplexy, hypnagogic/hypnopompic hallucinations and sleep paralysis. This frightening onset of symptoms often occurs in childhood or adolescence with a 10-14 year diagnostic delay. Often multiple misdiagnoses such as hypothyroidism, depression and epilepsy are made along the way. By presenting these patients, the features of narcolepsy are highlighted enabling Paediatricians to consider this as a diagnosis. Better recognition and earlier diagnosis can lead to earlier onset of therapeutic intervention thus leading to a lower impact on academic performance and social development.

\section{G70(P) RADIOLOGICAL INVESTIGATIONS OF MIDLINE INFANTILE HAEMANGIOMAS OVERLYING THE SCALP OR SPINE: 5 YEAR SINGLE CENTRE EXPERIENCE}

NV Cartledge, VA Kinsler, AE Martinez. Paediatric Dermatology, Great Ormond Street Hospital, London, UK

\subsection{6/archdischild-2015-308599.69}

Aim To review the outcomes of investigations for midline infantile haemangiomas (IH) overlying the head or spine in a tertiary paediatric dermatology department.

Methods Case notes and radiological results were reviewed retrospectively for all patients with a clinical diagnosis of $\mathrm{HI}$ overlying the midline of the scalp, neck or spine seen over the 5 year period October 2009-14.

Results 43 patients were identified (31 girls). The median age at the first specialist clinic appointment was 5 months (range 2-23 months). In 26 patients the $\mathrm{IH}$ were located on the scalp, in five on the back of the neck, in four overlying the thoracic spine and in eight overlying the lumbar spine and or sacrum. 24 lesions were more than $5 \mathrm{~cm}$ and 19 less than $5 \mathrm{~cm}$ in size at the time of the first appointment, 10 of which were plaque-type IH. 18/ 43 patients had MRI of either the brain or spine following the initial assessment. MRI was normal in 15 patients, including in all lumbosacral lesions. In two cases of plaque-type IH a diagnosis of PHACES syndrome was confirmed. In one child where the $\mathrm{IH}$ was a palm-sized lesion over the thoracic spine, MRI showed extension of the IH into the spinal canal, with complete compression of the cord between T4 and T9. The child was neurologically asymptomatic at the first assessment, but the result prompted treatment with propranolol.

Conclusion This retrospective series of midline IH seen over a five year period in a tertiary centre has identified a significant underlying lesion in a child with a thoracic spinal IH. The current literature from experts in the field recommends MRI for lumbosacral lesions. Our internal guidelines will now dictate an 